\title{
Hooliganismus in Deutschland
}

\section{Phänomenologie, Abgrenzung zu den „Ultras“, Drittortauseinandersetzung, Erklärungsansätze und Prävention}

Gabriele Kett-Straub

\section{Einleitung}

\section{Zahlen}

Zuerst die gute Nachricht: Deutschlands Fußballstadien sind sicher. Die jüngsten Vorfälle beim Relegationsspiel zwischen Fortuna Düsseldorf und Hertha BSC Ber$l_{i n}{ }^{1}$, im DFB-Pokal ${ }^{2}$ oder Anfang des Jahres bei einem Hallenfußballturnier in Hamburg sind (noch) die Ausnahme ${ }^{3}$. Anders als bspw. in Italien braucht man hierzulande auch Familien mit Kindern nicht vom Besuch eines Spiels ihres Vereins abzuraten. Ein Ausflug auf das Oktoberfest ist vermutlich gefährlicher ${ }^{4}$.

Nun die schlechte Nachricht: Die Zahl der in und um die Stadien verletzten Personen, die von der Polizei erfasst wurden, hat mit 846 in der 1. und 2. Bundesliga in der Saison 2010/11 einen neuen Höchststand erreicht (Saison 2009/10: 784 Personen) $^{5}$; die Zahlen der jetzt abgelaufenen Saison 2011/12 werden erst Ende des Jahres veröffentlicht. Diese Angaben stammen von der Zentralen Informationsstelle für Sporteinsätze (ZIS), die, angesiedelt bei der Landespolizei NRW, die Bundes- und Regionalligen seit Jahren auswertet. Hinzukommt ein - vermutlich aber konstant - großes Dunkelfeld, denn viele Schlägereien im Fußballkontext finden unter Gleichgesinnten ohne Anzeigebereitschaft statt.

Aufschluss über Fußballgewalt können aber auch die Zahlen der Deutschen Bahn geben, denn immerhin zwei Drittel aller Taten, die in Zügen und Bahnhöfen registriert werden, passieren im Zusammenhang mit Großveranstaltungen, insbesondere Fußballspielen. Gewalt und Sachbeschädigungen im Bereich der DB sind 2011 indes signifikant zurückgegangen ${ }^{6}$.

Laut Einschätzung der ZIS ist jedenfalls das Gewaltpotential der Fußballanhänger in der Saison 2010/11 nicht gestiegen, sondern es stagnierte auf hohem Niveau. Gewisse Schwankungen in den letzten Jahren haben eher damit zu tun, dass bestimmte Vereine ab- und andere aufsteigen. Die Zunahme der Gewalttaten speziell in der 2. Bundesli- ga in der vorletzten Saison erklärt sich fast ausschließlich durch den damaligen Abstieg von Herta BSC Berlin. Ein Aufstieg in die 1. Liga von BFC Dynamo Dresden oder gar Hansa Rostock - beide bekannt für ihre eher problematischen Fans - würde daher nicht nur Freudentränen hervorrufen ${ }^{7}$.

Man schätzt derzeit die Anzahl der gewaltbereiten Fußballanhänger in den beiden Profi-Ligen auf 9.685 Personen (diese Zahl setzt sich aus Fans der Kategorie B und C zusammen). Zur Erläuterung: Die Einteilung der Fußballfans durch die ZIS erfolgt in die Kategorien A, B und C, wobei „A“ der friedliche, „B“ der gewaltbereite/-geneigte und „C “ der gewaltsuchende Fan ist ${ }^{8}$. In der Verbunddatei „Gewalttäter Sport“ (GTS) sind gegenwärtig 13.032 Personen erfasst (Stand: 9.3.2012) ${ }^{9}$. Bei rund 18 Millionen Zuschauern, die pro Saison die Spiele der ersten beiden Ligen besuchen, ist dies in der Gesamtschau keine beeindruckende Größenordnung, aber auch keineswegs ein Anlass zur Beruhigung, wenn man bedenkt, welche Dynamik eine Gewalttat in einem ausverkauften Fußballstadion entwickeln kann. Die Ausschreitungen im Brüsseler Heysel-Stadion 1985 mit 39 Toten und fast 500 Verletzten haben uns dies in drastischer Weise vor Augen geführt ${ }^{10}$. Ein längerfristiger Vergleich zeigt aber sogar, dass die Zahl der wirklich gewaltsuchenden Fans (Kategorie C) über die Jahre leicht gefallen ist (Saison 1999/2000: 2.560 Personen; Saison 2010/11: 2.445). Evident gestiegen ist indes die Zahl der gewaltgeneigten Anhänger (1999/2000: 4.245 Personen; Saison 2010/11: 7.240) ${ }^{11}$. Dass Hooligangewalt aber vor Jahren noch viel radikaler war, ist fast schon vergessen: Am 4.11.1990 hat die (Volks-)Polizei in Leipzig auf randalierende Fans sogar scharf geschossen, weil sie sich nicht mehr anders zu helfen wusste. Ein Hooligan starb, einige andere wurden schwer verletzt ${ }^{12}$. Von solchen Zuständen ist man heute weit entfernt.

\section{2. Öffentliche Wahrnehmung}

Der Blick der Öffentlichkeit auf das Thema Fußballgewalt ist aber derzeit von Angst geprägt, wenn man zumindest die Berichterstattung in den Medien zugrunde legt. Da heißt es: „Die Randalier werden immer radikaler"13. Diese verzerrte Wahrnehmung der Sicherheitslage mag auch mit einer „stigmatisierenden Darstellung der Fankultur" zu tun haben ${ }^{14}$. So wird z.B. das vom Deutschen Fußball-Bund (DFB) in den Stadien verbotene Abbrennen von Pyrotechnik gleichgesetzt mit körperlichen Übergriffen. Einzelne Vorkommnisse werden zudem medial hochgespielt; viele hundert friedliche Fußballspiele sind dagegen keine Nachricht wert.

Außerdem gilt es zu bedenken, dass Ausschreitungen bei Sportveranstaltungen keineswegs ein neueres Phänomen darstellen. Explizit von Hooliganismus ist zwar erst seit den 1960er Jahren die Rede ${ }^{15}$, doch von Gewalt unter Zuschauern wird bereits seit den Anfängen des Sports berichtet ${ }^{16}$. Sport und Gewalt ziehen sich in gewisser Weise an; bei Fußball und Gewalt ist dies sogar in besonderem Maße der Fall. Nichtsdestotrotz muss vor der Gefahr einer Zuspitzung der Lage in und um die Fußballstadien in den kommenden Jahren gewarnt werden. Die Situation ist angespannt; die Stimmung emotional überfrachtet. Verantwortlich sind hierfür auch die Vereine und der DFB selbst, die Fußball als aufwändige Unterhaltungsshow inszenieren, um die vielen Millionen Zuschauer in die Stadien und vor allem vor die Bildschirme zu locken. Etwas mehr Nüchternheit statt überbordender Emotionalität wäre derzeit notwendig, doch dies würde dem Milliardengeschäft Fußball schaden.

\section{Begriffe}

Im folgenden Beitrag soll ein Überblick über das Phänomen Hooliganismus gegeben, Erklärungsansätze aufgezeigt und ein auf Hooligans maßgeschneidertes Präventionsmodell entwickelt werden. Auch die umstrittene Strafbarkeit der Drittortauseinandersetzungen, also verabredeten Kämpfen zwischen zwei gegnerischen Hooligangruppen abseits der Stadien, wird genauer untersucht werden. Unter dem Begriff Hooliganismus 
werden gewalttätige Ausschreitungen anlässlich von Fußballspielen verstanden ${ }^{17}$. Der Begriff ist insofern unscharf, als im Weiteren ausdrücklich zwischen den Fan-Gruppierungen Hooligans und Ultras unterschieden wird, Fußballgewalt aber generell unter der Überschrift Hooliganismus firmiert. Eine Differenzierung ist jedoch wichtig, wird aber auch in vielen wissenschaftlichen Untersuchungen nicht unternommen. Dies ist ein grundlegendes Versäumnis, denn die Motivation für Gewalt ist jeweils eine völlig andere und daher muss auch ein Präventionsmodell für die jeweilige Gruppe komplett unterschiedlich angelegt werden.

\section{Abgrenzung Hooligans/Ultras ${ }^{18}$}

\section{Die Ultra-Bewegung}

Fußball ist keine Sache von Leben und Tod. Er ist wichtiger als das. "19

Fußballanhänger sind keine homogene Gruppe, sondern es existiert sogar ein ausgesprochenes Kastendenken. Fälschlicherweise werden die Ultras oft in eine Schublade mit den klassischen Hooligans geworfen, da sie, was den Einsatz von Gewalt anbelangt, auch nicht von schlechten Eltern sind. Eine belastbare Definition des neueren Ultra-Phänomens gibt es nicht. „ExtremFan“ oder „Fußballanhänger mit Leib und Seele“, der sein Leben ganz dem Fan-Sein verschreibt, kommt dem Selbstverständnis eines Ultras nahe. Einen eingefleischten Fußballfan stellt man sich herkömmlicherweise in kompletter Montur in Vereinsfarben vor. Doch diese „Kuttenträger“ - benannt nach ihren mit Vereinsemblemen bestickten Jeanswesten - sind in den Stadien fast schon zum nostalgisch anmutenden Auslaufmodell geworden ${ }^{20}$. Schon seit einigen Jahren haben die Ultras die Vorherrschaft in den Fankurven übernommen ${ }^{21}$.

Die Ultragruppierungen sehen sich als die Wahrer einer neuen Fußballkultur. Sie haben sich zum Ziel gesetzt, den Fußball zu entkommerzialisieren. Daher tragen sie außer einem Schal bewusst keine als geschmacklos empfundenen Fanabzeichen, sondern meist schlichte schwarze, neuerdings auch orangefarbene Kapuzenshirts („,casual-style“) ${ }^{22}$, was eine optische Unterscheidung mit den Hooligans erschwert, die sehr ähnlich auftreten. Nach dem Vorbild italienischer Gruppen - Italien ist das Ursprungsland der Ultras - drücken sie ihre Verbundenheit mit ihrem Verein beim Spiel mit aufwändigen Choreografien und mit von einem "Сapo“ vorgegebenen Dauergesängen aus, auch wenn es bei der eigenen Mannschaft gerade schlecht läuft: Bedingungsloser „Support“ heißt die Devise.

Als Beispiel seien die beiden Nürnberger Ultra-Gruppierungen genannt, die auf den Stehplätzen - noch gibt es solche ${ }^{23}$ - in der Nordkurve des Stadions beheimatet sind. Sie nennen sich Ultra Nürnberg 94 - wer aufmerksam durch Nürnberg geht, sieht auf vielen Wänden „UN 94“ gesprüht - und banda di amici, eine kleinere Abspaltung, denen die Gründungsultras zu lasch agierten. Gemeinsam setze man sich für die Umbenennung des ehemaligen „easyCredit-Stadions" in ein sponsorenfreies "Max-Morlock-Stadion “ in Erinnerung an Nürnbergs bessere Fußballzeiten ein.

\section{Subkultur}

Das Phänomen Ultra zieht viele ganz junge Fußballanhänger in den Bann. Dennoch passt die Bezeichnung "Jugendsubkultur“ nur in einem sehr weiten Verständnis, denn zum einen ist das Altersspektrum der Ultras recht groß - auch wenn die Mehrheit der Mitglieder zwischen 15 und 25 Jahren alt ist ${ }^{24}$. Definiert man Subkultur als weitgehend isoliertes Milieu mit eigenen homogenen Werten und Normen in Abgrenzung zur bürgerlichen Gesellschaft, träfe dies wohl zum anderen auf die Ultra-Bewegung ebenfalls nur bedingt zu, denn die Verbindung zur Gesellschaft wird nicht gekappt. Dennoch fühlen sich viele Ultras von der Gesellschaft ausgegrenzt und haben Anpassungsprobleme.

\section{Gewaltbereitschaft}

\section{a) Sportorientierung}

Während bei Hooligans die gewalttätige Auseinandersetzung mit anderen Gruppen im Vordergrund steht, geht es bei den Ultras um den Sport. Dennoch ist bei vielen UltraGruppierungen Gewalt ein akzeptiertes Mittel zur Interessensdurchsetzung. So kann das „Erobern“ von gegnerischen Fanutensilien, etwa der Zaunfahne als Herzstück einer Ultra-Gruppierung, heftige Schlägereien auslösen. Beschwert sich ein unorganisierter Fußballzuschauer darüber, dass die Fahnen der Ultras ihm die Sicht versperren, wird er zumindest deutlich von ihnen eingeschüchtert. Aus der Befehlsgewalt der Ultras über den Fanblock kann also leicht körperliche Gewalt werden. Aggression kann außerdem eine Folge erlebter Frustration nach einem verlorenen Spiel sein; für die Entstehung von Hooligangewalt ist dagegen der Spielausgang unerheblich.

\section{b) Feindbild Polizei}

Ein weiteres wichtiges Thema der Ultras ist der Protest gegen das als Willkür und Repression empfundene Vorgehen von Polizei und Ordnern. In Sprechchören erklingt ihr unsäglicher Schlachtruf „All Cops are Bastards!“ oder „Fußballfans sind keine Verbrecher!“25. Das Verhältnis zwischen Polizei und Ultras kann man gelinde gesagt als belastet bezeichnen. Die Polizei ist der „zentrale Feind“ der Ultras ${ }^{26}$. Doch deren Verhalten kann - anders als das der Hooligans - noch gelenkt werden. Fußballgewalt ist im Fall der Ultras oft nur das spontane Ergebnis einer misslungenen Kommunikation ${ }^{27}$. Eine gelungene Fan-Polizei-Interaktion im Vorfeld kann Gewalt verhindern. Die hysterisch geführte Bengalo-Debatte ist dagegen das Musterbeispiel einer misslungenen Kommunikation. Die Fußballverantwortlichen stellten den Ultras in Aussicht, in den Stadien genehmigte Räume zum kontrollierten Abbrennen von Pyrotechnik zu schaffen ${ }^{28}$. Dieses Pilotprojekt wurde indes noch vor seinem Beginn vom DFB für gescheitert erklärt. Die Ultras fühlten sich an der Nase herumgeführt.

\section{c) Gefahr der Radikalisierung}

Die Situation ist seitdem noch angespannter und kann kippen, denn die Gefahr der Radikalisierung der Ultras ist vorhanden ${ }^{29}$. Ein Überfall von Mitgliedern der Kölner UltraGruppierung „Wilde Horde 1996“ auf einen Fanbus des Erzrivalens Borussia Mönchengladbach am 4.3. dieses Jahres muss indes noch als echtes Ausnahmeereignis und nicht als eine weitere Stufe hin zu einer Eskalation von Gewalt eingeschätzt werden. Die teilweise vermummten Ultras haben auf einem Rastplatz bei Siegburg die Scheiben des Busses mit Pflastersteinen eingeschlagen und versucht, das Fahrzeug auf der Autobahn A 3 mit ihren Autos auszubremsen und abzudrängen. Die Polizei durchsuchte daraufhin am 15.3. Privatwohnungen von Mitgliedern der Wilden Horde sowie deren Vereinsheim und stellte Waffen sicher ${ }^{30}$. Doch alle bundesweiten Ultra-Gruppierungen - einzelne Gruppen sind bis zu 1.000 Personen stark ${ }^{31}$ - machen der Polizei zunehmend Sorge, denn sie werden zwar mehrheitlich noch in die Fan-Kategorie „A“ eingestuft, aber die Aggressivität dieser Fans würde von Saison zu Saison steigen ${ }^{32}$. Man spricht daher bereits von „Hooltras“. Doch 
noch bleibt es meist bei „Scheinangriffen“ auf die Polizei oder die privaten Sicherheitsdienste $^{33}$. Schätzungen gehen aber davon aus, dass 20 bis $25 \%$ der Ultras irgendwann zu den Hooligans überlaufen ${ }^{34}$. Die Grenzen zwischen beiden Gruppierungen sind schon jetzt fließend. Gröbste Grenzziehung wäre, die Gruppen danach einzuteilen, wer noch in den Stadien ist.

\section{Hooligans}

\section{Definition}

Laut Definition der Bundesregierung versteht man hierzulande unter dem Begriff Hooligans „Personen, die im Zusammenhang mit Fußballbegegnungen durch Aggression und Gewaltbereitschaft auffallen und den Kontakt zu Gleichgesinnten des gegnerischen Vereins zur körperlichen Auseinandersetzung suchen “35. Streng genommen passen viele Hooligans daher auch nicht mehr unter die Kategorie „Fußballanhänger", wenngleich sie sich selbst als treue Fans bezeichnen würden ${ }^{36}$. Die Hooligans ${ }^{37}$ - zur Herkunft des Begriffs werden verschiedene Deutungen angeboten; vermutlich hat er sich aus dem Eigennamen prügelnder Iren entwickelt - sind nicht nur deshalb nicht in den Stadien, weil sie Stadionverbot haben ${ }^{38}$. Vielmehr interessiert sie das Fußballspiel nur noch am Rande. Der Spieltag setzt eher den zeitlichen und örtlichen Rahmen, sich mit gegnerischen Hooligangruppen zu vorab abgesprochenen „matches“ im privaten Städteturnier zu treffen. Hooligans (miss-) brauchen den Fußball als Anlass zur Gewaltausübung ${ }^{39}$.

\section{Merkmale}

Fast alle nationalen, aber auch internationalen Studien beschränken sich auf relativ kleine, lokale Gruppen ${ }^{40}$. Auch eine vom Bundesministerium des Inneren beauftragte Erhebung kann nur mit einer sehr kleinen Stichprobengröße aufwarten; befragt wurden u.a. von Lösel 33 Hooligans des „Harten Kerns" (man hat zwischen diesem und dem „Weiteren Kreis“ einer Gruppe unterschieden; ein spezielles Augenmerk wurde außerdem auf die Anführer gelegt $)^{41}$. Die Kritik lautet, dass insbesondere hierzulande über Hooligans letztlich mehr geschrieben als systematisch geforscht wird. Dieser Vorwurf ist nur eingeschränkt berechtigt. Zwar ist das Datenmaterial in der Tat vergleichsweise dünn, doch die Täterszene ist auch sehr schwer zugänglich und größere Vorhaben sind daher nur schwer realisierbar.
Hooligans sind im Schnitt älter als Ultras; doch das Altersspektrum ist erneut breit und reicht von etwa 16 Jahren bis Mitte/ Ende 40. Sie stammen aus allen sozialen Schichten ${ }^{42}$; die Zusammensetzung einer Gruppe ist regional sehr verschieden. Bildungsferne und Arbeitslosigkeit prägen die Szene, sind aber eher nicht die verbindenden Merkmale $^{43}$. Oft würden Hooligans aus der Türsteher- und Fitnessszene stammen ${ }^{44}$. Eine (Rand-)Erscheinung ist das Phänomen auffallend teuer gekleideter Hooligans ${ }^{45}$; es ist die Rede vom Wohlstandshooliganismus $^{46}$. Oft wird von Hooligans berichtet, die unter der Woche ein völlig bürgerliches Leben führen und nur am Wochenende ihr Aggressionsbedürfnis ausleben. „Gewalt ist eine Lösung“ ist der Titel eines Buches, in dem ein Polizist sein Doppelleben als Hooligan und Beamter beschreibt ${ }^{47}$. Es finden sich auch Ärzte oder Rechtsanwälte unter den Schlägern ${ }^{48}$, doch letztlich gibt es keine belastbaren Daten zur Anzahl solcher Doppelidentitäten und das Phänomen wird zu einem gewissen Teil auch reine Legendenbildung der Szene selbst sein. Hooligans sind in der Masse eher unpolitisch; die Schnittmenge bspw. mit Neonazis ist nur eine klei$n e^{49}$. Gemeinsamer Nenner ist die Affinität zu Gewalt. Rechtsradikale Äußerungen sind in der Hooliganszene austauschbares Provokationsmittel; in den 1970er Jahren haben sie noch „Es lebe die RAF!" skandiert ${ }^{50}$.

Die Gruppe („firm“) selbst ist keine situative Tätergemeinschaft, sondern es finden sich durchaus komplexe Strukturen. So gibt man sich im Sinne einer kollektiven Identität bspw. einen Namen (Bsp: „GelsenSzene“, „Elbflorenz Dresden“ oder „Adler Front Frankfurt" $)^{51}$. Es gibt aber keine echte Hierarchie ${ }^{52}$; die Anführer sind eher dazu da, die „dates“ zu verabreden (hierzu sogleich). Kriminologische Kategorien des Täterzusammenschlusses im Sinne von Bandenkriterien erfüllt höchstens der harte $\mathrm{Kern}^{53}$; den weitaus größeren Teil der Gruppe stellen aber die Mitläufer (weicher Kern).

\section{Gewalt}

\section{a) Drittortauseinandersetzung}

Spontane oder abgesprochene Hooligangewalt richtet sich in der Regel gegen andere Hooligangruppierungen oder die Polizei. Der starke Gruppenbezug kennzeichnet die Gewaltausübung ${ }^{54}$; von Deindividuationseffekten kann daher ausgegangen werden. Hooligans können aber in komplett videoüberwachten Stadien ihrem Hauptstreben, dem „fight“, nicht mehr ungestraft nachgehen. Ihre private Fußball-Schlacht wird daher in die weniger gut gesicherten unteren Ligen verlagert oder findet gar völlig abseits der Stadien statt. Man verabredet am Spieltag mit den Gegnern per Handy oder E-Mail ein „date“ an ruhigen und verlassenen Orten, in Wäldern, auf Feldern oder in Gewerbegebieten.

Das „match“ selbst läuft nach einem festen Schema ab. Beide Mannschaften stehen sich in der "dritten Halbzeit“ in etwa gleich starken Gruppen von 20 bis 40 Mann gegenüber und stürmen bei Ertönen eines Signals zum Kampf aufeinander zu. Die „Guten“ stehen in erster Reihe und gehen keinem Fight aus dem Weg. Die Mitläufer heißen im Szenejargon „Lutscher“ und kommen mit, um die „Guten“ zu bewundern. Frauen sind nicht teilnahmeberechtigt. Man prügelt nur wenige Minuten. Dann sammelt man sich zum nächsten Durchgang. Die reine „Lehre“ schreibt vor, dass man nicht auf die Genitalien des Gegners tritt. Erlaubt ist nur Passivbewaffnung wie Mundschutz, Schienbeinschoner, Handschuhe oder Sturmhauben. Jegliche Schlag-, Hieb- und Stichwaffen sind verboten. Es darf nur leichtes Schuhwerk getragen werden. Man kämpft nur Mann gegen Mann und liegt ein Gegner am Boden, lässt man von ihm ab. Flüchtende Gegner werden nicht verfolgt. Ein Schiedsrichter überwacht das Kampfgeschehen.

Letztlich ist aber unklar, wie eng sich die Szene an ihren Ehrenkodex gebunden fühlt ${ }^{55}$. Ein Verweisen auf ein solches Regelwerk von Seiten der Hooligans hat vermutlich vor allem mit Neutralisierungstechniken zu tun, da sie ihre Gewalttätigkeiten selbst gerne zu fairen, sportlichen Wettkämpfen hochstilisieren möchten. Üblicherweise startet ein Hooligan laut den Selbstauskünften mit Prellungen, Stauchungen, Hämatomen, Platz- und Schürfwunden, ausgekugelten Fingern oder einem Rippenbruch in die neue Woche. Schwerere Verletzungen können vermutet werden; sie würden aber nicht zur Anzeige gebracht werden.

\section{b) Strafbarkeit}

\section{aa) Landfriedensbruch}

Die strafrechtliche Relevanz der „FeldWald-Wiese "-Treffen ist umstritten; zur Debatte stehen Landfriedensbruch gem. \ 125 Abs. 1 Nr. 1 StGB und Körperverletzungsdelikte. Voraussetzung des Landfriedensbruchs ist einmal, dass Gewalttätigkeiten aus einer Menschenmenge heraus 
mit vereinten Kräften begangen werden. Strafgrund der Vorschrift ist, der Gefahr einer Masseneskalation entgegenzuwirken, aber wohl auch die Umgehung der Beweisnot bei aus der Masse heraus begangenen Straftaten ${ }^{56}$. Grundsätzlich ist der Landfriedensbruch kein ausschließliches Demonstrationsdelikt ${ }^{57}$; die Subsumtion einer vorab geplanten Drittortauseinandersetzung unter $\ 125 \mathrm{StGB}$ wäre daher keineswegs von vornherein ausgeschlossen.

Wann eine Menschenmenge i.S. der Vorschrift vorliegt, kann nicht klar beziffert werden. Laut Rspr. würden bspw. 10 Personen für die Annahme einer solchen noch nicht ausreichen, sofern nicht noch weitere Umstände hinzutreten würden (Unübersichtlichkeit des Tatorts etc. $)^{58}$. Bei einer typischen Drittortauseinandersetzung kann aber davon ausgegangen werden, dass die erforderliche Mindestanzahl der Teilnehmer erreicht wird. Die Gewalttätigkeiten müssen außerdem aus dieser Menschenmenge heraus begangen werden und nicht nur innerhalb derselben. Stehen sich aber genügend große Teilgruppen einer Menschenmenge in gewalttätiger Absicht gegenüber, kann die erforderliche Außenwirkung angenommen werden $^{59}$. Eine „normale“ Bierzeltschlägerei könnte daher bei entsprechender „Teilnehmerzahl“ zum Landfriedensbruch wer$\operatorname{den}^{60}$.

Allerdings könnte die Strafbarkeit des Landfriedensbruchs vor allem am rein privaten Hintergrund der Drittortauseinandersetzung mangels Gefährdung der öffentlichen Sicherheit scheitern ${ }^{61}$. Dieses schwer zu konturierende Merkmal setzt nach h.M. voraus, dass für eine unbestimmte Anzahl von Personen oder Sachen die Gefahr eines Schadens eintritt ${ }^{62}$. Darüber hinaus soll es auch genügen, wenn durch die Tat das Sicherheitsgefühl unbestimmt vieler Menschen beeinträchtigt wird ${ }^{63}$. Zu fragen sei insbesondere danach, ob der Eindruck entstehen muss, dass man in unserem Gemeinwesen nicht mehr frei vom Terror gewalttätiger Mengen leben kann ${ }^{64}$. Dies würde man auch bei Individualangriffen nur dann verneinen können, wenn diese lediglich den Charakter einer vorwiegend „privaten“ Auseinandersetzung haben. Die Handlungen müssten gleichsam unter Ausschluss der Öffentlichkeit stattfinden ${ }^{65}$. Als Beispiel für ein tatbestandsfremdes Geschehen wird in der Kommentarliteratur gerne das sich prügelnder, rivalisierender Rockerbanden genannt ${ }^{66}$. Doch diese Konstellation entspricht gerade nicht einer typischen
Drittortauseinandersetzung ${ }^{67}$. Vielmehr verabreden sich die Hooligans zum Kampf zwar abseits der Stadien, aber immer auf öffentlichen Grund im Kontext eines höchst öffentlichkeitswirksamen Fußballspiels. Eine relative Privatheit, die zum Ausschluss der Vorschrift führen müsste, ist daher (in der Regel) nicht anzunehmen. Um das Rockerbeispiel der Kommentare nochmals aufzugreifen: Würden die Bandidos gegen die Hells Angels auf öffentlichen Grund zum Kampf antreten, würde sich kaum jemand gegen die Annahme eines Landfriedensbruchs aussprechen.

Zudem wäre eine Gefährdung der öffentlichen Sicherheit im Fall der Hooligans (wie auch der Rocker) spätestens dann zu bejahen, wenn die Polizei eingreift und sich vermutlich selbst tätlichen Angriffen ausgesetzt sehen wird. Dagegen kann nicht eingewandt werden, würden die Beamten sich nicht einmischen, würden sie sich auch nicht in Gefahr begeben. Vielmehr sind diese sowohl aufgrund ihrer präventiven als auch repressiven Aufgaben zum Tätigwerden verpflichtet. Deren Handlung wird quasi von den Hooligans herausgefordert, um einen zivilrechtlichen Gedanken einfließen zu lassen. Im Übrigen wäre aber die Subsidiaritätsklausel des Abs. 1 zu beachten, die nach strittiger Ansicht auch bei Körperverletzungsdelikten zum Tragen kommt ${ }^{68}$; mit \ 113 StGB würde die Vorschrift dagegen in Tateinheit stehen ${ }^{69}$.

\section{bb) Körperverletzung}

Die aktive Teilnahme an einer Drittortauseinandersetzung beinhaltet begriffsnotwendigerweise das Begehen einer Körperverletzung i.S. von $\$ 223$ StGB. Je nach Fallgestaltung sind außerdem auch $\$ 224$ Abs. 1 Nr. 4 StGB oder Nr. 2 bzw. bei Eintritt der entsprechenden Folgen auch $\$ 231$ Abs. 1 StGB einschlägig. Fraglich ist aber, ob die Hooligans (sofern sie sich noch im Bereich der SS 223, 224 StGB bewegen) rechtswidrig handeln. Schließlich willigen die Teilnehmer des Schlagabtausches in mögliche Verletzungen gemäß $\$ 228$ StGB ein, so dass zwar nicht die Tatbestandsmäßigkeit ${ }^{70}$, doch aber die Rechtswidrigkeit entfallen könnte. Allerdings besteht bei einem Hooligan-Fight immer die hohe Wahrscheinlichkeit des Mitwirkens Minderjähriger und für deren Einwilligung müssten schon erhebliche Zweifel an einer hinreichenden Selbstbestimmungsfähigkeit geäußert werden ${ }^{71}$.
Außerdem könnte die Wirksamkeit der Einwilligung an ihrer Sittenwidrigkeit scheitern. Dies wäre der Fall, wenn die Tat, in die eingewilligt wird, gegen die guten Sitten - also das Sittlichkeitsempfinden aller billig und gerecht Denkenden - verstößt ${ }^{72}$. Im Bereich der Körperverletzungen beschränken BGH und herrschende Literatur die normative Wertung über $\$ 228 \mathrm{StGB}$ auf Art und Gewicht des Körperverletzungserfolges $^{73}$. Die Schwelle zur Sittenwidrigkeit ist unstreitig jedenfalls dann überschritten, wenn die konkrete Gefahr des Eintritts eines schweren Körperverletzungserfolges i.S. der $\mathbb{\int} \int 226,227$ StGB besteht ${ }^{74}$. Einen besonders großzügigen Maßstab legt man aber für Verletzungen im Sport und speziell für Kampfsportarten an, die auf gravierende Verletzungen geradezu abzielen ${ }^{75}$. Die Gefahr der schweren Gesundheitsschädigung und somit der Sittenwidrigkeit werde durch die Sozialadäquanz bspw. von Boxkämpfen aufgewogen, also durch die allgemeine Überzeugung, dass dieses sportliche Kräftemessen seinen gesellschaftlichen Wert hat ${ }^{76}$.

Diese Prinzipien könnten aufgrund der selbstgesetzten Regeln auch auf die „Wettkämpfe" der Hooligans angewendet wer$\operatorname{den}^{77}$. Doch der Vergleich einer Drittortauseinandersetzung mit einer Sportveranstaltung hinkt: Ein Hooligan-Fight ist gerade kein sozialadäquater Wettkampf, wie dies etwa bei einem regelkonformen, von einem der Verbände organisierten Boxkampf der Fall wäre ${ }^{78}$. Die Ähnlichkeit der Verletzungserfolge und auch die Tatsache, dass schwere Folgen angeblich nicht zu erwarten seien, vermögen an dieser Einschätzung nichts zu ändern. Wenn das $A G$ Esslingen und in der Folge das LG Stuttgart eine Drittortauseinandersetzung als eine Art „Mannschaftskickboxen“ bezeichnen und daher von einer Straflosigkeit der Beteiligten ausgehen, ist das eine fast schon naiv anmutende Verharmlosung der Situation ${ }^{79}$. Wer könnte die Einhaltung des angeblich strengen Reglements durchsetzen, würde die Lage eskalieren? In der Befragung von Lösel war zwar allen Hooligans der Ehrenkodex bekannt, doch nur ein kleiner Teil fühlte sich auch wirklich an ihn gebunden ${ }^{80}$. Dagegen verstießen drei Viertel der Befragten nach eigenem Bekunden häufig oder gelegentlich gegen das Regelwerk. Immerhin ein Drittel dieser Hooligans bejahte auch den Einsatz von Waffen wie Schlagringen oder Baseballschlägern. Spätestens dann wird auch der Einsatz eines Szene-Schiedsrichters nur mehr wenig ausrichten können. Im Ergebnis ist daher in der Regel von der Sittenwidrig- 
keit der Einwilligung auszugehen und eine Strafbarkeit wegen Körperverletzungsdelikten eröffnet ${ }^{81}$. Bezüglich der angeblichen Ungefährlichkeit solcher Kämpfe sollte man sich eher nicht auf die von Neutralisierungsmechanismen geprägten Selbstauskünfte der Akteure verlassen; auch aus der Szene stammendes Videomaterial ist als Beweismittel nur bedingt geeignet.

\section{Rolle der Medien}

Ausführliche Berichterstattung über Krawalle wird von der Szene erwünscht und hat Belohnungscharakter. Für Hooligans ist ihre Medienpräsenz ein wichtiger Beweggrund; Ultras fühlen sich dagegen von der Presse im Zweifel missverstanden. Die Fights werden außerdem von den Mitläufern gefilmt und - gerne mit Musik unterlegt - in geschlossen Internetforen veröffentlicht oder auch auf der Internet-Plattform „youtube“ eingestellt. Beklebte früher ein Hooligan ein Album mit Zeitungsberichten, sammelt er heute auf einem USB-Stick seine größten Erfolge. Vergleichbares ist nur von den Graffiti-Sprayern bekannt. „Fame“ ist das, was es in beiden delinquenten Szenen gleichermaßen zu erreichen gilt ${ }^{82}$. Von einem Interdependenzverhältnis zwischen der in den Massenmedien gefundenen Aufmerksamkeit und einer Wiederholung oder Nachahmung der Taten kann ausgegangen werden ${ }^{83}$. Dennoch sollte die Rolle der Medien auch nicht mystisch im Sinne einer ursächlichen Verantwortlichkeit überbewertet werden.

\section{Erklärungsansätze}

Die traditionellen Kriminalitätstheorien, insbesondere die Aggressionstheorien, aber auch vorwiegend schichtbezogene Ansätze, helfen als Erklärungsmuster für das Phänomen Hooliganismus nur bedingt weiter. Eine Frustrations-Aggressions-Hypothese im Hinblick auf das Verlieren der eigenen Mannschaft ist insofern schon fragwürdig, als - wie dargestellt - Hooligangewalt unabhängig vom Spielausgang stattfindet ${ }^{84}$. Gerade die Führungspersonen einer Gruppe sind keine sozialen Außenseiter, die ihren Frust über diese Gesellschaft abreagieren wollen. Hooliganismus ist auch nur in einem gewissen Umfang klassengebunden, sondern vorwiegend individualistisch ${ }^{85}$. Erklärungsansätze aus Großbritannien, wonach Hooliganismus eine Reaktion der Arbeiterklasse und als Widerstand gegen die Mittelschicht zu verstehen wäre, können nicht auf deutsche Verhältnisse übertragen werden $^{86}$. Zumindest eine gewisse Anzahl der Hooligans lebt hierzulande unter der Woche sogar - wie dargestellt - ein rundum bürgerliches Leben. Devianz am Wochenende und angepasstes Alltagsverhalten schließen sich nicht aus.

Im Unterschied zu den Ultras spielt bei den Hooligans auch Alkohol bei der Entstehung von Gewalt kaum eine Rolle ${ }^{87}$, was nicht heißt, dass danach nicht Alkohol im großen Umfang konsumiert wird ${ }^{88}$. In den Selbstberichten der Hooligans ist jedenfalls davon die Rede, dass man im Fight nüchtern sein müsse. Die Einnahme aufputschender Drogen vor Hooligan-Kämpfen scheint indes verbreitet $^{89}$.

Nach der von Heitmeyer/Peter entwickelten Entwertungstheorie (basierend auf der Beckschen Individualisierungsthese) ist das Phänomen Fangewalt durch die „Schattenseiten gesellschaftlicher Individualisierungsprozesse" verursacht ${ }^{90}$. Demnach würde die moderne Gesellschaft dem Einzelnen mit erhöhten Bildungschancen und einem Anstieg des Lebensstandards zwar erheblich mehr Möglichkeiten bieten, gleichzeitig gingen aber traditionelle Bindungen verloren. Für den Jugendlichen würde diese Verlust an sozialem Halt bei gleichzeitiger Erweiterung der Freiheiten häufig zu sozialer Desintegration führen. Im Fußballkontext würde zudem die zunehmende Kommerzialisierung dieses Sports mit der einhergehenden Enttäuschung über die fehlende Bindung zum Verein zu einem Verstärker dieses Auflösungsprozesses werden. Die Ausübung von Gewalt würde dagegen eine innere Ordnung schaffen können. Doch auch diese These stellt ausschließlich den Jugendlichen in den Mittelpunkt einer Betrachtung und würde eher das Phänomen der Ultras darstellen können. Hooligangewalt ist aber nicht jugendzentriert; es bleibt erneut ein Erklärungsdefizit.

Neben dieser rein soziologischen Erklärung wird vor allem die Zivilisationstheorie von Elias als Begründung des Phänomens Hooliganismus herangezogen ${ }^{91}$. Demnach würde die offene körperliche Gewalt mehr und mehr aus unserer zivilisierten Gesellschaft verbannt werden, doch der einzelne Mensch könne mit dieser Entwicklung noch nicht mithalten. Er brauche vielmehr Ventile, um seine aufgestauten Bedürfnisse ausleben zu können. Der Fußball würde ihm in diesem „Wechselspiel der gesellschaftlichen Entwicklungen " den nötigen sozialen Freiraum bieten können ${ }^{92}$.

\section{Sensation Seeking}

„Der Reiz liegt in dem Moment, wenn du um die Ecke biegst und 40 Mann auf dich zurennen. Das ist der Kick für den Augenblick. Das ist wie Bungee-Springen - nur ohne Seil. "93

\section{Persönlichkeitsmerkmal}

Gewalt und Aggression sind immer multifaktoriell bedingt und die Prozesse, die zu ihrer Entstehung führen, stehen wiederum mit vielfältigen gesellschaftlichen Prozessen in Wechselwirkung ${ }^{94}$. Selbstverständlich sind auch die sozialen Hintergründe der einzelnen Hooligans bei diesen Erwägungen nie zu vernachlässigen, auffällig oben ist doch, dass die Gewalt der Hooligans weder spontan, noch eine Reaktion ist. Zur Ursachenforschung ist man daher vorwiegend auf situationsunabhängige Gründe angewiesen. Die Protagonisten fühlen sich eher wie moderne Gladiatoren. Hooliganismus hat Eventcharakter. Von den Hooligans wird auch als erlebnisorientierten in Abgrenzung zu den konsum- bzw. sportorientierten Fußballanhängern gesprochen ${ }^{95}$.

In dieser Konsequenz ist im Fall der Hooligans ein Erklärungsmodell besonders weiterführend: Menschen unterscheiden sich in ihrem Bedürfnis nach Stimulation. Der Psychologe Marvin Zuckermann beobachtete bei manchen Probanden ein besonders ausgeprägtes Verlangen nach Nervenkitzel und Angst-Lust-Erfahrung ${ }^{96}$. Man geht davon aus, dass es für jeden Menschen ein optimales Erregungsniveau gibt, das Zwillingsstudien zufolge sogar eine gewisse genetische Varianz hat. Das Persönlichkeitsmerkmal des "Sensation Seeking“ ist demnach ein physiologisch begründetes Konstrukt und wurde bislang vor allem im Kontext mit Spielsüchtigen genauer untersucht ${ }^{97}$. Interessant ist in diesem Zusammenhang, dass es zwischen dem Pathologischen Spielen und dem Hooliganismus Parallelen gibt, etwa was die gestörte Impulskontrolle anbelangt ${ }^{98}$.

Menschen mit dem Persönlichkeitsmerkmal „Sensation Seeking“ scheinen bspw. zwar nicht grundsätzlich anfälliger für Drogenkonsum, aber anfälliger speziell für Heroin zu sein, also eine Droge, von der weniger Experimentierfreudige von vornherein die Finger lassen würden. Besonders ausgeprägt ist die ständige Suche nach Reizen und Abwechslung bei jungen Männern ${ }^{99}$. Typische Sensation-Seeker üben bspw. sozial akzeptierte Aktivitäten wie Fallschirmspringen 
oder Tiefseetauchen aus. Die erlebten Reize wirken auf Sensation-Seeker kurzfristig sehr belohnend; es erfolgt indes eine relativ schnelle Abnutzung ${ }^{100}$. Seine persönliche Ausprägung kann man anhand des Fragenkatalogs der von Zuckermann entwickelten Sensation Seeking Scale (SSS) einschätzen $^{101}$.

Berichten Hooligans von ihren Fights, fällt auf, dass es weniger um den Kampf selbst geht, sondern um den Moment davor, „das Angst haben“. Sie berichten vom Tunnelblick und dem Adrenalinstoß vor einer Auseinandersetzung ${ }^{102}$. Hooliganismus wird als Versuch beschrieben, zu spüren, dass man lebt ${ }^{103}$. Ziel ist der Rausch ohne Drogen, wenngleich teilweise sogar eine nichtstoffgebundene Abhängigkeit beobachtet wird ${ }^{104}$. Zentrales Motiv dieser Personengruppe, die außerdem eine allgemeine Disposition zur Aggression zeigt und auffällig oft sogar psychopathische Züge im Sinne der Psychopathy-Checklist von Hare aufweist (dies gilt vor allem für die Anführer ${ }^{105}$, ist der „Kick“ in Form der Kombination eines Gruppenerlebnisses mit Gewalt. Im 1992 erschienenen Buch "Among the Thugs" (deutscher Titel: Geil auf Gewalt. Unter Hooligans) des amerikanischen Journalisten Bill Buford lässt sich eindrucksvoll nachvollziehen, wie der Autor, der britische Hooligans auf Fahrten zu Auslandsspielen begleitete, seinen anfänglichen Ekel vor deren Brutalität überwindet und der Faszination Hooligan letztlich selbst erliegt.

\section{Erlebniskriminalität}

Übersetzt in die Kriminologie kann diese Reizsuche Erklärungsmodell für die sogenannte Erlebniskriminalität sein ${ }^{106}$. Die Gewalttätigkeit ist in diesem Fall schlicht Freizeitgestaltung und nicht von einem konkreten Anlass abhängig. Hooliganismus ist letztlich das abwegige Hobby der Akteure ${ }^{107}$. Hochproblematisch wird dieses Bedürfnis nach „Thrill“ auch deshalb, weil es mit Gewöhnungseffekten und dem eben erwähnten Suchtpotential einherzugehen scheint ${ }^{108}$. Das bedeutet, dass der Fußballanhänger mit dem Persönlichkeitsmerkmal „Sensation Seeking“, der einmal Blut geleckt hat - um ein zwar martialisches, aber letztlich treffendes Bild der Situation zu zeichnen - nur schwerlich wieder aufzuhören vermag. Er zeigt ein dranghaftes Verlangen nach dem erstrebten Effekt ${ }^{109}$. Diese Erkenntnis schmälert die Erfolgsaussichten vieler Präventionsmaßnahmen von vornherein deutlich.

\section{Prävention/Intervention}

Gewaltbereite, erlebnisorientierte Personen verhalten sich von vornherein konfrontativ und Lösungsansätze abseits von Kontrolle und Repression sind letztlich wenig erfolgsversprechend. Wenn man bei Hooligans sogar Symptome einer nichtstoffgebundenen Abhängigkeit vom „Kick“ und psychopathische Züge beobachten kann, darf man davon ausgehen, dass bspw. Gefährderansprachen im Vorfeld durch die Polizei oder Fan-Projekte, die die Bindung zum Verein wieder stärken sollen, wenig bewirken werden. Dies gilt jedenfalls für den harten Kern einer Gruppe. Bezüglich des weiteren Kreises einer Hooligan-Gruppe mag klassische Fanarbeit dagegen durchaus eine wirksame Maßnahme darstellen können. Das Ziel müsste sein, diesen Personenkreis vom harten Kern abzuspalten und ihn wieder in die Stadien als echte Fans zu bekommen. Hauptzielgruppe der Fanprojekte sollten indes die Ultras sein, deren Bezug zum Verein und deren aus falsch verstandener Solidarität mangelnde Bereitschaft, Gewalttäter in ihren Reihen auszusondern, es dringend zu stärken gilt.

Die Lust an ihren Handlungen ist für Hooligans auch deshalb so groß, weil diese kriminell sind. Die Polizei als Lieblingsgegner auf Augenhöhe wertet ihre Aktionen auf und wird daher bewusst in diese postmodernen Cowboy- und Indianerspiele eingebunden. Würde die Polizei sich zurückziehen, was speziell für die Drittortauseinandersetzungen gefordert wird, wäre das ganze Szenario nur halb so reizvoll. Die Hooligans müssten wieder näher an die Stadien heranrücken, um die Polizei herauszufordern. Nicht erfolgversprechend ist auch das vereinzelt vorgeschlagene „Wiesenmodell“, also die behördliche Bereitstellung von Flächen für die Fights. Ein solches Konzept wäre genauso zum Scheitern verurteilt, wie Graffiti-Sprayern eine offizielle Hauswand für ihre Bilder zur Verfügung zu stellen ${ }^{110}$.

Empfohlen werden muss daher, dass die Polizei den Hooligans gegenüber deutliche Präsenz zeigen muss. Der harte Kern der Hooligans muss von szenekundigen Beamten pausenlos am Spieltag begleitet werden und es sollten aufenthaltsbeschränkenden Maßnahmen im Vorfeld einer Fußballveranstaltung ausgesprochen werden. Ferner helfen wohl nur eine niedrige Einschreitschwel- le und eine konsequente Strafverfolgung, die Gewalt einzudämmen. Das vorhandene Instrumentarium ist indes ausreichend.

Da insbesondere die Anführer einer Gruppe deutliche Tendenzen zur antisozialen Persönlichkeitsstörung zeigen, sollte außerdem über eine Behandlungsbedürftigkeit dieser Gruppe und Therapieangebote nachgedacht werden. Echte Prävention müsste indes deutlich früher ansetzen. Lösel schlägt diesbezüglich verschiedene nicht stigmatisierende Programme vor, etwa solche zur Stärkung der Erziehungskompetenz der Eltern, Anti-Gewaltprogramme in der Schule oder das Training sozialer Kompetenzen für Kinder ${ }^{111}$. Viel stärker müsste aber in allen Überlegungen die Erkenntnis berücksichtigt werden, dass manche Menschen mehr als andere auf der Suche nach extremen Reizen in ihrem Leben sind. In einer Gesellschaft, deren Streben nach Sicherheit immer ausgeprägter und die gleichzeitig immer gefahrloser wird, muss dem Einzelnen die Möglichkeit verbleiben, sein persönliches Bedürfnis nach Risiko auszuleben. Wie das im Einzelnen umgesetzt werden kann, muss überlegt werden. Statt langweiligen Schulsports könnte Box- oder Fechtsport für die notwendige Abwechslung sorgen. Klettergärten könnten in Pausenhöfen entstehen und selbst die umstrittenen Paintball/Gotcha-Spielfelder könnten auf der Suche nach Nervenkitzel Abhilfe schaffen.

\section{Fazit}

Das Gewaltpotential der Fußballanhänger stagniert auf hohem Niveau. Hooligans haben ihre gewalttätigen Auseinandersetzungen angesichts der umfangreichen Präventionsmaßnahmen zwar weg von den Stadien verlagert, doch mit einem Erliegen des gesellschaftlichen Phänomens Hooliganismus ist nicht zu rechnen. Gewalt und Aggression ist zwar immer multifaktoriell bedingt, doch als Erklärungsmuster helfen rein schicht- oder altersbezogene Ansätze nur bedingt weiter. Die Gewalttätigkeit der Hooligans ist Freizeitgestaltung und nicht von einem konkreten Anlass abhängig; sie ist Erlebniskriminalität. Zentrales Motiv dieser Personengruppe, die von vornherein eine allgemeine Disposition zur Aggression hat, ist das „Kick-Erlebnis“. Die Bedeutung des besonderen Stimulationsbedürfnisses des "Sensation Seeking“ ist unter den vielen Erklärungsansätzen für Hooliganismus herausragend. Nur eine Wechselwirkung zwischen einem vielleicht schwierigen Sozialisationsverlauf und dieser speziellen Persön- 
lichkeitsdisposition vermag das Phänomen Hooliganismus annähernd zu erklären. Präventionsarbeit müsste demnach unbedingt das Bedürfnis der Akteure nach Abenteuer berücksichtigen.

Aktuell hilft in und um die Stadien vor allem eine Null-Toleranz-Strategie der Behörden, um die Gewaltausschreitungen von klassischen Hooligans einzudämmen. Das eigentliche Problem ist, dass ein solches Vorgehen nur bei dieser speziellen Klientel erfolgsversprechend ist. Andere Fangruppierungen, insbesondere die Ultras, empfinden schon die bloße Anwesenheit der Polizei als Provokation, auf die es ihrerseits mit Gewalt zu reagieren gilt. Die Forderungen an diese Fans dürfen aber nicht überstrapaziert werden. Die große Herausforderung ist daher, eine Balance der Maßnahmen zu finden. Muss man für Hooligans die Räume eng machen - um im Fußballjargon zu bleiben - müssen auf der anderen Seite den Ultras unbedingt Freiräume belassen werden ${ }^{112}$.

Die Autorin ist Privatdozentin am Institut für Strafrecht, Strafprozessrecht und Kriminologie an der Friedrich-Alexander-Universität Erlangen-Nürnberg. Stand der Internetquellen: 13.6.2012.

\section{Fußnoten:}

1 Das Spiel am 15.5.2012 musste zwischenzeitlich abgebrochen werden, nachdem Fans in der Nachspielzeit das Spielfeld stürmten und Feuerwerkskörper (Bengalos) auf den Platz geschossen wurden.

2 Bei der Partie Dynamo Dresden gegen Borussia Dortmund (0:2) am 25.10.2011 in Dortmund haben randalierende Dresdner Anhänger fast für einen Spielabbruch gesorgt und einen hohen Sachschaden verursacht. Als Konsequenz schloss das DFB-Sportgericht Dresden von der Teilnahme am DFB-Pokal 2012/13 aus. Bei der Partie zwischen Eintracht Frankfurt und dem 1. FC Kaiserslautern (0:1) am 26.10.2011 waren mindestens 8 Polizisten verletzt worden.

3 Rivalisierende Fan-Gruppen der Clubs VfB Lübeck, FC St. Pauli und Hamburger SV lieferten sich bei einem Hamburger Hallenfußballturnier am 6.1.2012 so heftige Prügeleien, dass die Veranstaltung abgebrochen werden musste.

4 Beispiel übernommen aus einem Beitrag in der FAZ v. 13.11.2011, online verfügbar unter: http://www.faz.net/-gtm-6v2z3.

5 Jahresbericht Fußball der Saison 2010/11, Landesamt für Zentrale Polizeiliche Dienste NRW (LZPD NRW), ZIS, S. 8: Zusammensetzung der verletzten Personen: 243 Polizeibeamte, 259 Störer und 344 Unbeteiligte; online verfügbar unter: http://www.polizei-nrw. de/media/Dokumente/10-11Jahresberichtoeffentlich.pdf.

6 Die Zahl der Angriffe auf Bahnpersonal ist indes gestiegen; vgl. Sicherheitsbericht der DB v. 15.11.2011, online verfügbar unter: http:// www.deutschebahn.com/de/presse/presseinformationen/pi_k/2312352/h20120307a. html. Der Rückgang der Körperverletzungen in Zügen und an Bahnhöfen ist zumindest auch mit verbesserten Sicherheitskonzepten und dem vermehrten Einsatz von Personal zu erklären.

7 Laut ZIS gelten 130 Fans von Hansa Rostock als "gewaltbereit" und 390 als "gewaltgeneigt“. Am 18.12.2011 wurden daher bei einem Heimspiel von Hansa Rostock gegen Dynamo Dresden in der 2. Bundesliga keine Zuschauer zugelassen. Der DFB hat das „Geisterspiel“" als Strafe ausgesprochen, weil es am 19.11.2011 rund um das Duell mit dem FC St. Pauli zu schweren Ausschreitungen gekommen war. Damals hatten Hansa-Fans mit Leuchtraketen auf Zuschauer aus Hamburg geschossen und versucht, in den Gästeblock einzudringen. Speziell zu dem Sicherheitskonzept für die Dresdner Fanszene siehe Pätzold/ Kaempf, Kriminalistik 2012, 217 ff.

8 Vgl. Jahresbericht Fußball der Saison 2010/11 (o. Fn. 5), S. 5.

9 BT-Drucks. $17 / 9003$ v. 16.3.2012. Es werden beim BKA die Daten von Personen gespeichert, gegen die im Zusammenhang mit Sportveranstaltungen wegen bestimmter Straftaten (Katalog [u.a. $\$ \mathbb{S} 113,315$ ff., 240, 123, 185, 86a, 130 StGB] und Generalklausel: „Straftaten unter Anwendung von Gewalt gegen Leib oder Leben oder fremde Sachen mit der Folge eines nicht unerheblichen Sachschadens") zumindest ein Ermittlungsverfahren eingeleitet wurde. Darüber hinaus können die Daten von Personen erfasst werden, wenn bestimmte Tatsachen die Annahme rechtfertigen, dass sie künftig Straftaten von erheblicher Bedeutung im Zusammenhang mit Sportveranstaltungen begehen werden. Ein Eintrag kann bspw. Grundlage für Ausreiseverbote bei internationalen Turnieren oder für Meldeauflagen sein. Die von den Medien sogenannte „Hooligan“-Datei stieß bei den Untergerichten aber zunehmend auf Kritik, da es an einer Rechtsgrundlage mit klaren Kriterien fehlte. Zwischenzeitlich wurde nachgebessert und am 9.6.2010 ist eine entsprechende Verordnung des Bundesinnenministeriums in Kraft getreten, so dass das BVerfG (BVerwGE 137, 113) nun die Speicherung der Daten als rechtmäßig eingestuft hat.

10 Am 29.5.1985 anlässlich des Endspiels um den Pokal der Landesmeister 1984/85 zwischen dem FC Liverpool und Juventus Turin stürmten Anhänger Liverpools in den neutralen Sektor. Es brach Panik aus und eine Wand stürzte ein.

11 BT-Drucks. $17 / 8051$ v. 2.12.2011, S. 3 und Jahresbericht Fußball der Saison 2010/11 (o. Fn. 5), S. 5.

12 Vgl. Spiegel Online v. 12.11.1990, online verfügbar unter: http://www.spiegel.de/spiegel/ print/d-13503293.html. Vgl. auch Farin/Seidel-Pielen, Krieg in den Städten, 1991, S. 92.

13 Z.B. „Randalierer in Fußballstadien werden immer radikaler", Beitrag im Berliner Tagesspiegel v. 18.12.2011, online verfügbar unter: http://www.tagesspiegel.de/sport/randaliererin-fussballstadien-werden-immer-radikaler/5971570.html.

14 Gabriel, Fußballfans sind keine Verbrecher. Das schwierige Verhältnis zwischen Polizei und Fanprojekten, in: Möller (Hrsg.), Dasselbe in grün? Aktuelle Perspektiven auf das Verhältnis von Polizei und Sozialer Arbeit, 2010, S. $47 \mathrm{ff}$.

15 Albrecht, MschrKrim 89 (2006), 158.

16 Beispiele aus der Antike (Stock- und Peitschenträger sorgten in Olympia für Ordnung unter den Zuschauern) und weiterführende Nachw. bei Pilz, Fußball und Politik, 1/2006, online verfügbar unter: http://www.buergerimstaat.de/1_06/tatort.htm.

17 Siekmann, Fußball-Hooliganismus, in: Walker (Hrsg.), Hooliganismus, 2009, S. 61.
18 Aufgrund seiner italienischen Herkunft wird das Wort „Ultrà “ teilweise mit Akzentzeichen geschrieben.

19 Das Zitat stammt angeblich von der Trainerlegende des FC Liverpool William „Bill“ Shankly.

20 „Neckermänner" werden normale Fußballzuschauer genannt; vgl. Meier, Hooliganismus in Deutschland, 2001, S. 59.

21 Weiterführend Gabler, Die Ultras: Fußballfans und Fußballkulturen in Deutschland, 2011; Sommerey, Die Jugendkultur der Ultras, 2010; Langer, Faszination Ultras, 2010 und Brenner, Neues aus der Fankurve - Wie Ultras und andere Fangruppierungen die Fankultur verändern, 2009. Die UltraBewegung entstand im Italien der frühen 1950er und 1960er Jahre, als sich erstmals „fußballverrückte“ Jugendliche in Gruppen zusammenschlossen, um ihre jeweiligen Lieblingsmannschaften gemeinsam organisiert zu unterstützen. Der Name der Bewegung geht angeblich auf eine italienische Zeitung zurück, die Anhänger des FC Turin als „Ultrà “ bezeichnete, als diese nach einem Spiel einen Schiedsrichter aus Wut bis zum Flughafen verfolgten.

22 Angesichts des breitgefächerten Angebots an Merchandising-Artikeln für Ultras im Internet zweifelt man allerdings an der Ernsthaftigkeit der Entkommerzialisierung. Im Übrigen verfügen die Ultra-Gruppierungen über eigene Internetauftritte (z.B. http://www.un94.com) und eine bundesweite Zeitschrift „Blickfang Ultra" (auf der Titelseite des werbefreien Novemberheftes zeigt ein Mann seinen mit den Worten "Scheiß auf Arbeit - Lebe Ultra“ tätowierten Rücken; vgl. http://www.blickfangultra.de/). Die einzelnen Gruppen werden teilweise hierarchisch von einer "Direttivo“ von 10 bis 15 Personen geführt; andere (kleinere) Gruppen funktionieren basisdemokratisch.

23 Die Münchner Allianz Arena hat bspw. 56.000 Sitz- und 13.000 Stehplätze (bei internationalen Begegnungen gibt es von vornherein nur Sitzplätze). Maßgeblich aus Sicherheitsgründen wird die Anzahl der Stehplätze - sehr zum Leidwesen der Ultras - in vielen Stadien immer mehr reduziert; über eine komplette Umwandlung aller Steh- in Sitzplätze wird diskutiert. Experten warnen vor dieser Entwicklung, die die Ultras als „Kampfansage“ verstehen würden; vgl. Interview in der Frankfurter Rundschau v. 18.3.2010 mit Michael Gabriel, Leiter der vom Bund und DFB finanzierten Koordinierungsstelle Fanprojekte (KOS), online verfügbar unter: http://www.fr-online.de/sport/ interview-mit-michael-gabriel-die-ultras-machen-sorgen,1472784,3130738.html.

24 Ziegler, Gewalt im Zusammenhang mit Fußballspielen - Sind italienische Verhältnisse auch in Deutschland denkbar?, in: Sport und Gewalt, Schriftenreihe der Deutschen Hochschule der Polizei III-IV, 2009, S. 59 (100).

25 Gerne verwendet wird auch auf T-Shirts das Kürzel A.C.A.B. bzw. „Acht Cola, acht Bier“ mit jeweils fettgedruckten Anfangsbuchstaben. In Nürnberg wird immer ein Verfahren wegen Beleidigung eröffnet; eine etwas gelassenere Sichtweise der Behörden würde das Verhältnis Polizei/Ultras verbessern können.

26 Pilz, Deutsche Polizei, 11/2005, 6 ff., online verfügbar unter: http://www.buergerimstaat. de/1_06/tatort.htm.

27 Feltes, neue praxis (np) 4/2010, 405 (415 ff.).

28 Spiegel Online v. 9.11.2011; online verfügbar unter: http://www.spiegel.de/sport/ fussball/0,1518,druck-796667,00.html.

29 Pilz, Kriminalistik 2012, 203 (207 f.); vgl. auch Gabriel in einem Interview in der Zeitschrift 11 Freunde - Magazin für Fußball- 
kultur v. 29.12.2009; online verfügbar unter: http://www.11freunde.de/interview/kos-leiter-michael-gabriel-ueber-fan-gewalt.

30 Nach Polizeiauskunft war die Razzia am 15.3.2012 der erste Einsatz dieses Ausmaßes; vgl. spiegel-online v. 15.3.2012, online verfügbar unter: http://www.spiegel.de/panorama/justiz/0,1518,821574,00.html.

31 BT-Drucks. $17 / 8051$ v. 2.12.2011, S. 5. Versuch einer Schätzung auch bei Gabler, Die Ultras, 2011, S. 58. 2006 schätzten Pilz/Wölkli die Anzahl der Ultras auf 6.000 Personen, vgl. Ultraszene in Deutschland, in: Pilz u.a. (Hrsg.), Wandlungen des Zuschauerverhaltens im Profifußball, 2006, S. $63 \mathrm{ff}$.

32 Vgl. Jahresbericht Fußball der Saison 2010/11 (o. Fn. 5), S. 6; Ziegler (o. Fn. 24), S. 59 ff.

33 So beispielsweise beim Lokalderby des 1. FC Nürnberg gegen die SpVgg Greuther Fürth (0:1) am 20.12.2011, als frustrierte Nürnberger Ultras das Spielfeld stürmten.

34 Vgl. Bericht „Ultras, Hooligans, Hooltras“ auf spiegel-online v. 21.6.2007; http://www. spiegel.de/sport/fussball/0,1518,488211,00. html.

35 Vgl. BT-Drucks. 17/8051 v. 2.12.2011 (Antwort der Bundesregierung auf eine Kleine Anfrage der Fraktion „Die Linke“).

36 Die Mehrzahl der Hooligans gibt an, Zugang zu der Szene über ihr bereits in der Kindheit gewecktes Fußballinteresse gefunden zu haben; Lösel/Bliesener, MschrKrim 89 (2006), 229 (236).

37 Der Begriff Hooligans (engl. Schlägertypen, Raufbolde, Rabauken) geht angeblich au eine irische Familie namens O'Houlihan zurück, die sich im 19. Jahrhundert wegen ihrer Prügeleien einen derart üblen Ruf erworben hat, dass sie sogar in Trinkliedern besungen wurde. Es werden aber noch weitere Erklärungen angeboten; vgl. Meier (o. Fn. 20), S. 9.

38 Laut Zentralstelle des DFB für die Erfassung bundesweit wirksamer Stadionverbote sind derzeit 2.994 solcher jeweils zweijähriger Verbote in Kraft (Stand: 22.2.2012); vgl. BTDrucks. $17 / 9003$ v. 16.3.2012. Seit der Saison 1999/2000 sind insgesamt 9.527 Stadionverbote ausgesprochen worden; vgl. BT-Drucks. $17 / 8051$ v. 2.12 .2011 , S. 4. Zur RechtmäBigkeit der Stadionverbote vgl. BGH, Urteil v. 30.10.2009 - V ZR 253/08, BeckRS 2009, 29234 = JZ 2010, 249. Ein Bayern-Fan, dessen Körperverletzungsverfahren nach \$ 153 StPO eingestellt worden war, hatte sich vergebens gegen das dennoch ausgesprochene Hausverbot gewehrt. Doch laut BGH sei ein solches Verbot gegen einen Fußballanhänger dann vom Hausrecht des Veranstalters gedeckt, sofern ein sachlicher Grund vorliege: „Ein sachlicher Grund besteht dann, wenn aufgrund von objektiven Tatsachen (...) die Gefahr besteht, dass künftige Störungen durch die betreffenden Personen zu besorgen sind (2. Leitsatz).

39 Böttger, Die Gewalt der Hooligans - eine Folge moderner gesellschaftlicher Entwicklungsprozesse, KFN-Forschungsberichte $\mathrm{Nr}$. 71, 1998, online verfügbar unter: http://www. $\mathrm{kfn} . \mathrm{de} / \mathrm{versions/kfn/assets/fb71.pdf.}$

40 Böttger hat bspw. zehn (jugendliche) Hooligans mit der Technik des narrativen Interviews befragt (o. Fn. 39).

41 Lösel/Bliesener/Fischer/Papst, Hooliganismus in Deutschland: Ursachen, Prävention und Intervention, Abschlussberichts eines Forschungsprojektes für das Bundesministerium des Innern, 2001, bezeichnen ihre Erhebung im Vergleich zu anderen aber „als vergleichsweise breit und intensiv angelegt", vgl. Lösel/ Bliesener, MschrKrim 89 (2006), 229 (230).

$42 \mathrm{Krahm}$, Polizeiliche Maßnahmen zur Eindämmung von Hooligangewalt, 2008, S. 57.
43 Lösel/Bliesener/Fischer/Papst (o. Fn. 41) verweisen auf deutliche Unterschiede in den verschiedenen Datenquellen, S. 149.

44 Ziegler (o. Fn. 24), S. 59 (110).

45 Teure Jacken der Marke „Chevignon“ waren ein früher gern gewähltes Bekleidungsstück dieser Gruppe. Heute kleidet man sich bei diversen Internetshops ein, die „HooliganStreetwear" in allen Preisklassen anbieten.

46 Gehrmann, Hooligans in Deutschland, 1990, S. 25

47 Schubert, Gewalt ist eine Lösung. Morgens Polizist, abends Hooligan, 2010.

48 Lösel/Bliesener/Fischer/Papst (o. Fn. 41) schränken insofern ein, als der „psychisch gesunde(r) Arzt oder Anwalt als Hooligan wohl die Ausnahme wäre", S. 163.

49 Vgl. auch BT-Drucks. 17/7962 v. 30.11.2011.

$50 \mathrm{Krahm}$ (o. Fn. 42), S. 57.

51 Schwind, Kriminologie, 21. Aufl. 2011, $\mathbb{2 8}$, Rdn. 29b.

52 Hartmann, Gewalt in der Gruppe, in: Kröber u.a. (Hrsg.) Handbuch der Forensischen Psychiatrie, Bd. IV, S. 209 (216 f.).

53 Wenngleich man in der Kriminologie den nach wie vor recht unbestimmten Bandenbegriff fast ausschließlich im Kontext der Jugendkriminalität diskutiert.

54 Krahm (o. Fn. 42), S. 53.

55 Lösel/Bliesener, MschrKrim 89 (2006), 229 (234)

56 Ostendorf, in: NK StGB, 3. Aufl. 2010, $\mathbb{} 125$ Rdn. 1

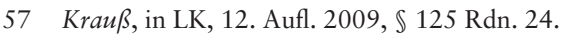
58 BGH NStZ 2002, 538.

59 Fischer, StGB, 59. Aufl. 2012, \$125 Rdn. 7.

60 BGH NJW 1986, 1116 mit Anm. Otto, NStZ 1986, 70

61 Spoenle, NStZ 2011, 552 (553 f.).

62 BGHSt. 18, 271 (272); Lackner/Kühl, 27. Aufl. 2010, $\$ 125$ Rdn. 9.

63 Schäfer, in: MK StGB, 2005, $\mathbb{1 2 5}$ Rdn. 19 m.w.N.

64 OLG Köln NStZ-RR 1997, 235.

65 Rackow, in: BeckOK StGB, Stand: 15.3.2012, $\$ 125 \mathrm{Rdn} .11$

66 Lenckner/Sternberg-Lieben, in: Schönkel Schröder, 28. Aufl. 2011, $\mathbb{1} 125 \mathrm{Rdn} .11$.

67 A.A. Spoenle, NStZ 2011, 552 (554).

68 BGHSt 43, 237; Schäfer, in: MK, \$ 125 Rdn. 48; a.A. Gesetzeskonkurrenz, da Landfriedensbruch nur gegenüber solchen Strafnormen zurücktreten würde, die im Wesentlichen dem Schutz derselben Rechtsgüter dienen (,relative Subsidiarität) vgl. Rudolphil Stein, in: SK StGB, 7. Aufl. 2004, \$ 125 Rdn. 28

69 Lackner/Kühl, $\mathbb{1 2 5}$ Rdn. 16.

70 Eine Einschränkung schon auf Ebene der Tatbestandsmäßigkeit wird aufgrund einer Sozialadäquanz von sportlicher Betätigung gelegentlich bei bestimmten Sportarten diskutiert, nicht aber bei solchen mit Verletzungsziel, genauer hierzu Dölling, ZStW 96 (1984) 36 ff. (55 ff., 64); a.A. nur Zipf, Einwilligung und Risikoübernahme im Strafrecht, 1970, S. 77 ff.; Zipf, ZStW 82 (1970) 633. Doch die Lehre, nachdem ein solches Einverständnis schon tatbestandsausschließend wirken könnte, setzt sich über den klaren Wortlaut des $\mathbb{2} 28$ StGB hinweg. Aus gleichem Grund wird auch die Möglichkeit eines erlaubten $R i$ sikos nicht weiterverfolgt.

71 Fischer, $\mathbb{} 228 \mathrm{Rdn} .5$

72 BGHSt. 4, 24 (32); BGHSt. 4, 88 (91).

73 BGHSt. 49, 34 (43); BGHSt. 49, 166 (169 ff.).

74 Für einen weiteren Anwendungsbereich plädiert überzeugend Hardtung, in: MK StGB,

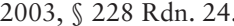

75 Speziell zum Boxsport vgl. Fritzweiler, SpuRt 1995, 156

76 Hardtung: in MK, 2003, $\$ 228$ Rdnr. 34.
77 Aktuell kommt zu diesem Ergebnis Spoenle, NStZ 2011, 552 ff. Zur Drittortauseinandersetzungen in der Rspr.: VG Stuttgart, Urt. v. 14.9.2009 - 5 K 2929/08, BeckRS 2010, 45133; Beschl. v. 8.6.2006 - 5 K 2106/6, BeckRS 2006, 25975; OVG Magdeburg, Beschl. v. 19.6.2006 - 2 M 216/06, BeckRS 2008, 32709.

78 A.A. AG Esslingen, Urt. v. 30.6.2008 - 3 Ds 2 Js 73015/05; nachfolgend auch LG Stuttgart, Urt. v. 8.12.2010 - 42 Ns 2 Js 73015/05.

79 AG Esslingen, Urt. v. 30.6.2008 - 3 Ds 2 Js 73015/05, S. 6; LG Stuttgart, Urt. v. 8.12.2010 - 42 Ns 2 Js $73015 / 05$, S. 11 . Kritisch Wagner, DÖV 2011, 234.

80 Lösel/Bliesener/Fischer/Papst (o. Fn. 41), S. 125.

81 Ein Verbots- bzw. Erlaubnistatbestandsirrtum mag je nach Tatkonstellation erwogen werden können; vgl.Spoenle, NStZ 2011, 552 (556); Fischer $\$ 228$ Rdn. 5.

82 Feltes, neue praxis (np) 4/2010, 409 m.w.N.

83 Eisenberg, Kriminologie, 6. Aufl. 2005, \$ 57 Rdn. 15; Krahm (o. Fn. 42), S. 53 f.

84 Denker, Angst und Aggression, 1974, S. 139.

85 Göppinger, Kriminologie, 6. Aufl. 2008, $\$ 28$ Rdn. 46.

86 Z.B. Dunning/Murphy/Williams, Zuschauerausschreitungen bei Fußballspielen - Versuch einer soziologischen Erklärung, in: Elias/Dunning (Hrsg.), Sport und Spannung im Prozeß der Zivilisation, 2003, S. 433 (449 ff.).

87 Anders bspw. die These von Albrecht, MschrKrim 2006, 158 (164). Insofern hätte ein oft gefordertes Alkoholverbot in den Stadien und in deren Nahbereich als Maßnahme zur Gewaltprävention im Hinblick auf die Hooligans - im großen Unterschied zu den Ultras - keine positive Auswirkung. Derzeit gelten unterschiedliche Promillegrenzen für den Einlass in ein Stadion; ein generelles Ausschankverbot für Alkohol gibt es nur in ganz wenigen Stadien bzw. bei „Risiko“-Spielen, vgl. hierzu einen Beitrag bei welt-online v. 14.10.2011, online verfügbar unter: http:// www.welt.de/sport/fussball/article13660856/ Promillegrenzen-spalten-die-Fussball-Bundesliga.html.

$88 \mathrm{Krahm}$ (o. Fn. 42), S. 58.

89 Lösel/Bliesener/Fischer/Papst (o. Fn. 41), S. 162.

90 Heitmeyer/Peter, Jugendliche Fußballfans. Soziale und politische Orientierung, Gesellungsformen, Gewalt, 2. Aufl. 1992, S. 9; Beck, Risikogesellschaft, 1986.

91 Elias, Über den Prozess der Zivilisation, Soziogenetische und psychogenetische Untersuchungen, Band 1, 1976, S. 312 ff., 322

$92 \mathrm{Krahm}$ (o. Fn. 42), S. 47 f.

93 Selbstauskunft eines Hooligans über seine Motivation; zitiert aus Pilz, Fußball und Politik, 1/2006, S. 8, online verfügbar unter: http://www.buergerimstaat.de/1_06/tatort. htm.

94 Lösel/Bliesener/Fischer/Papst (o. Fn. 41) sehen bei der Mehrheit der von ihnen interviewten Hooligans bereits erhebliche Schwierigkeiten in der Herkunftsfamilie, S. 162.

95 Heitmeyer/Peter (o. Fn. 90). Benutzt man den Begriff allerdings im Zusammenhang mit gewalttätigen Ausschreitungen, sieht man sich leicht dem Vorwurf der Verharmlosung ausgesetzt; vgl. spiegel-online v. 24.11.2011, online verfügbar unter: http://www.spiegel. de/sport/fussball/0,1518,799827,00.html.

96 Zuckerman, Sensation Seeking: Beyond the optimal Level of Arousel, 1979; ders., Biological Bases of Sensation Seeking, Impulsivity and Anxiety, 1983; ders., Sensation Seeking and Risky Behavior 2007.

97 Die biologische Basis des Konstrukts wird durch EEG-Studien gestützt. 
98 Lösel/Bliesener/Fischer/Papst (o. Fn. 41), S. 152.

99 Chuck Palahniuk beschreibt in seinem Roman „Fight Club“, 1996 (1999 verfilmt von David Fincher u.a. mit Brad Pitt), typische Sensation-Seeker. Es treffen sich Männer zu Kämpfen im Keller einer Bar. Diese Art von Geheimloge ist für den Protagonisten die neue Form einer Selbsthilfegruppe.

100 Kunczik/Zipfel, Gewalt und Medien, 2004, S. 51.

101 Bspw. mit Hilfe des deutschen Fragenkatalog von Gniech/Oetting/Brohl, Untersuchungen zur Messung von „Sensation-Seeking“, 1993.

102 Schubert, Gewalt ist eine Lösung. Morgens Polizist, abends Hooligan, 2010.
103 Farin, Von Guten und Mitläufern, online verfügbar unter: http://www.bpb.de/gesellschaft/ kultur/jugendkulturen-in-deutschland/36239/ von-guten-und-mitlaeufern.

104 Lösel/Bliesener/Fischer/Papst (o. Fn. 41), S. 151.

105 Hare, Psychopathy Checklist-Revised (PCLR), 1991 - deutsche Übersetzung von $\mathrm{Ne}$ dopil/Müller-Isberner;2001; vgl. auch Lösel/ Bliesener/Fischer/Papst (o. Fn. 41), S. 113.

106 Göppinger (o. Fn. 85), $\mathbb{2} 28$ Rdn. 46.

107 Von Erlebniskriminalität geht man auch bei Graffiti-Sprayern aus, Neubacher, ZStW 118 (2007), $855 \mathrm{ff}$.

108 Göppinger (o. Fn. 85), $\mathbb{S} 28$ Rdn. 46.
109 Lösel/Bliesener/Fischer/Papst (o. Fn. 41), S. 151.

110 Lösel/Bliesener, MschrKrim 89 (2006), 229 (239).

111 Lösel/Bliesener/Fischer/Papst (o. Fn. 41), S. 151.

112 Von den seit ca. einem Jahr von der Polizei praktizierten sogenannten „mobilen Massenkontrollen“ der Fußballfans auf Bahnhöfen ist daher unbedingt abzuraten (Züge auf dem Weg zu einem Spiel oder zurück werden angehalten und alle Fans zum Aussteigen aufgefordert, identifiziert und fotografiert), vgl. BT-Drucks. 17/8053 v. 2.12.2011.

\title{
Sicherungsverwahrung: Das Bundesverfassungsgericht als Erfüllungsgehilfe eines gehetzten Gesetzgebers?
}

\author{
Michael Bock/Sebastian Sobota
}

\section{Einleitung}

Die jüngere Rechtsprechung des Bundesverfassungsgerichts (BVerfG) zur Sicherungsverwahrung, insbesondere das Grundsatzurteil $^{2}$ vom Mai 2011, ist in der Literatur überwiegend positiv aufgenommen wor$\operatorname{den}^{3}$. Der Einschätzung, es handele sich bei dem Urteil um einen mutigen Befreiungsschlag, der ähnlich herausragend wie die Urteile bspw. zur lebenslangen Freiheitsstrafe oder zur gesetzlichen Grundlage des Strafvollzugs sei ${ }^{4}$, ist jedoch - wie sogleich zu zeigen sein wird - aus mehreren Gründen entschieden zu widersprechen. Vielmehr wecken die jüngeren Urteile und Beschlüsse zur Sicherungsverwahrung die Befürchtung, dass sich das BVerfG dem Druck von Öffentlichkeit und Politik beugt und vor den Karren punitiver Kriminalpolitik ${ }^{5}$ spannen lässt. Es geht um nicht weniger als die Frage, ob Straftäter noch denselben grundrechtlichen Schutz wie die „Normalbevölkerung" genießen.

II. Das sog. „Abstandsgebot“ - das StVollzG als lediglich symbolisches Recht?

Bereits der in der verfassungsgerichtlichen Argumentation zentrale Begriff des Abstandsgebotes, wonach sich die Ausgestaltung des Vollzugs der präventiven Sicherungsverwahrung qualitativ vom Freiheits- entzug durch Strafe unterscheiden müsse ${ }^{6}$, ist problematisch ${ }^{7}$. Mit dem Abstand zum Strafvollzug wählt das $B \operatorname{VerfG}$ nämlich einen falschen Maßstab. Tatsächlich kann es aufgrund der unterschiedlichen Legitimation mit Schuld auf der einen (Strafe) und dem Sonderopfer zugunsten der Allgemeinheit auf der anderen Seite (Sicherungsverwahrung) nicht auf den Abstand des Strafvollzugs gegenüber dem Maßregelvollzug ankommen, wie ihn insoweit die fehlplatzierten und überdies materiell unzureichenden ${ }^{8}$ Regelungen der $\$ \mathbb{S} 129 \mathrm{ff}$. StVollzG nahelegen. Wenn es nämlich bloß auf den Abstand zwischen Straf- und Maßregelvollzug ankäme, wäre die Verfassungsmäßigkeit des Vollzugs der Sicherungsverwahrung bereits ohne jede Verbesserung für die Verwahrten wieder hergestellt, wenn die Bedingungen im Strafvollzug (wieder) restriktiver bzw. resozialisierungsfeindlicher würden ${ }^{9}$.

Maßstab für die Ausgestaltung des Vollzuges der Sicherungsverwahrung muss (nicht nur aus kriminologischer Sicht) vielmehr das Leben in Freiheit sein ${ }^{10}$. Um es mit den Worten des Strafvollzugsgesetzes auszudrücken, muss „das Leben im Vollzug den allgemeinen Lebensverhältnissen [...] angeglichen werden “ ( $\int 3$ Abs. 1 StVollzG). Dies gilt für die Sicherungsverwahrung deshalb umso mehr, weil die Untergebrachten ihre Strafe verbüßt haben und rein präventiv zum Schutz der Allgemeinheit verwahrt werden. Einschränkungen in der Angleichung darf es somit nur aus absolut zwingenden Gründen der Sicherheit (und nicht aus ökonomischen, punitiven oder sonst praktischen Erwägungen heraus) geben ${ }^{11}$. Dies ist auch deshalb geboten, weil das Verhalten in der Unterbringung mit zunehmender Nähe zum Leben in Freiheit an prognostischer Aussagekraft gewinnt ${ }^{12}$.

Zum Widerspruch fordern auch Bewertungen heraus, nach denen das aus dem Abstandsgebot abgeleitete Vollzugskonzept des $B$ VerfG von „großer Sensibilität und Sachkunde“ 13 zeuge. Zusammengefasst lässt sich zwar sagen, die neue Sicherungsverwahrung soll nach dem Willen des $B \operatorname{VerfG}$ therapeutisch und freiheitsorientiert ausgestaltet sein, es soll gerichtlichen Rechtsschutz, Lockerungen sowie Entlassungsvorbereitung geben und es soll auch die Mitwirkungsbereitschaft der Untergebrachten gefördert werden. Doch zum einen scheint ein derart konkreter Gesetzgebungsauftrag durch ein Verfassungsgericht kaum mit dem Gewaltenteilungsprinzip vereinbar ${ }^{14}$. Zum anderen fällt hier sogleich auf, dass die Regelungen, die das $B$ VerfG für eine verfassungskonforme Ausgestaltung der Sicherungsverwahrung fordert, gar keine anderen sind als die des aktuell geltenden (Bundes-) 